\title{
The Effect of Near-Wall Vortices on Wall Shear Stress in Turbulent Boundary Layers
}

\author{
Shuangxi Guo ${ }^{1}$, Wanping $\mathbf{L i}^{2}$ \\ ${ }^{1}$ School of Civil Engineering and Mechanics, Huazhong University of Science and Technology, Wuhan, China \\ ${ }^{2}$ The Key Laboratory of Mechanics on Western Disaster and Environment of the Ministry of Education of China, \\ Lanzhou, China \\ Email: gsx 2001@163.com \\ Received June 10, 2009; revised July 9, 2009; accepted July 21, 2009
}

\begin{abstract}
The objective of the present study is to explore the relation between the near-wall vortices and the shear stress on the wall in two-dimensional channel flows. A direct numerical simulation of an incompressible two-dimensional turbulent channel flow is performed with spectral method and the results are used to examine the relation between wall shear stress and near-wall vortices. The two-point correlation results indicate that the wall shear stress is associated with the vortices near the wall and the maximum correlation-value location of the near-wall vortices is obtained. The analysis of the instantaneous diagrams of fluctuation velocity vectors provides a further expression for the above conclusions. The results of this research provide a useful supplement for the control of turbulent boundary layers.
\end{abstract}

Keywords: Spectral Methods, Two-Dimensional Turbulence, Wall Shear Stress, Two-Point Correlation

\section{Introduction}

The flow phenomenon of turbulent boundary layers is common in nature. It is closely related to aerospace, marine, environmental energy, chemical engineering and other fields. In aeronautical engineering, the complex turbulent vortex structures in boundary layers not only affect the working stability and security of the aircraft but also increase the skin friction on the wall remarkably. So the research of control of turbulent boundary layers is significant. In recent years the related literatures focus mainly on two aspects: control of near-wall turbulent structures and wall skin friction. Essentially, the wall skin friction is closely related with the near-wall turbulent structures, so the researches on these two aspects are in accordance. In flat wall flows, the wall shear stress constitutes wall skin friction. Sheng, Malkiel and Katz did much in-depth and complete study on the relation between wall shear stress (streamwise and spanwise) and near-wall flow structure (streamwise, spanwise and outside structure) by experiment method [1]. Most researchers agreed that the near-wall streamwise vortices were the main effect factors of wall shear stress [2-5]. The wall normal and spanwise velocities boundary conditions were presented by the methods of wall blowing and suction or spanwise-wall oscillation, which directly changed the near-wall streamwise vortices and achieved the purpose of control of the wall shear stress [6-8]. Though the detailed mechanism has not been completely clear so far, the above-mentioned control methods have made good effectiveness on wall shear stress reduction. Recently Y. S. Park et al also researched the control of wall shear stress by the method of wall blowing and suction. Instead of vertical to the wall, certain angles were presented between the blowing-suction direction and the streamwise direction. This meant that the velocity boundary conditions brought by their control method were normal and streamwise velocities instead of normal and spanwise velocities. Their experiments showed a better effectiveness of wall shear stress reduction if the angle was proper [9]. In fact, wall normal and streamwise velocity boundary conditions changed the near-wall spanwise vortices directly. As well as streamwise vortices, spanwise vortices are also the main characteristics in turbulent boundary layers, while are they also the crucial effect factors on wall shear stress as the streamwise vortices?

Essentially, turbulent flow is absolutely three-dimensional, but some certain turbulence motion, such as atmosphere or ocean flows, is behaving quasi-two-dimensionally. The horizontal scales are hundreds of kilometers in the ocean and thousands of kilometers in the at- 
mosphere, while their vertical scale is only a few kilometers. So the turbulent motions in the vertical direction are suppressed and can be ignored can be treated as twodimensional turbulence [10-11]. The saturation states of two-dimensional turbulence have the similar characteristics as the three-dimensional turbulence, such as injection, sweep and other bursting phenomenon [12-13], while they have lots of differences from the three-dimensional turbulence, such as self-organization and inverse energy cascade [10-11]. In addition, the simulation of two-dimensional turbulence requires less expensive computational resources in comparison with that of three-dimensional turbulence. So it is also valuable for the study of two-dimensional turbulence. Moreover, scalar vorticity in two-dimensional turbulence is controlled by the normal and streamwise velocity, and has the same expression as the spanwise vorticity in three-dimensional turbulence, $\eta=\frac{\partial v}{\partial x}-\frac{\partial u}{\partial y}$. So in the present paper the two-dimensional scalar vorticity is taken as the major subject of study instead of the three-dimensional spanwise vorticity. The objective of the present study is to explore the relation between the near-wall vortices and the shear stress on the wall in two-dimensional channel flows.

So far, the researches of two-dimensional turbulence are mainly limited to the models with unbounded condition, or with the identical bounded condition such as square or circular domains. The literatures of two-dimensional channel flow are rare. W. Kramer, H. J. H. Clercx and G. J. F. van Heijst have done some pioneering study on this subject. They have researched the influence of the aspect ratio of the channel and the integral-scale Reynolds number on the large-scale self-organization of the flow in detail and obtained lots of important consequence [11]. In the present paper, the numerical process to simulate the two-dimensional turbulent channel flows directly with spectral method is firstly introduced, and the accuracy and stability of the proposed algorithm is verified with two examples. Secondly the relation between the wall shear stress and near-wall vortices is explored and the maximum correlation-value location of the near-wall vortices is obtained. Finally the instantaneous diagrams of fluctuation velocity vectors and the near-wall model are analyzed to provide a further expression for the conclusions obtained.

\section{Numerical Processes}

\subsection{Numerical Method}

With the development of computational technology and resources, the direct numerical simulation (DNS) is more and more widely used as the basic research approach of turbulence. Spectral method is one of the most common methods for the DNS of turbulence, which has many advantage, such as high degree of accuracy, quickly speed on convergence, and analytically spatial derivation for flow variables [13]. Many researchers have done lots of pioneering and significant achievements for this method, such as John Kim, Moin \& Moser [14], Kleiser \& Schumann [15], Hu, Morfey \& Sandham [16]. So spectral method is applied to solve the Navier-Stokes equation directly in the present study.

The governing equations for two-dimensional incompressible channel flow can be written as the following forms:

$$
\begin{aligned}
\frac{\partial u_{i}}{\partial t}+f_{i} & =-\frac{\partial p}{\partial x_{i}}+\frac{1}{\operatorname{Re}} \nabla^{2} u_{i} \\
\frac{\partial u_{i}}{\partial x_{i}} & =0
\end{aligned}
$$

Here, all variables are non-dimensionalized by the channel half-width $\delta$ and laminar Poiseuille flow central velocity $U_{c}$; Non-linear term $f_{i}$ includes the convective terms and the mean pressure gradient [14]; and Re denotes the Reynolds number defined as $\operatorname{Re}=U_{c} \delta / v$, where $v$ is kinematic viscosity.

Vorticity $\eta$ is defined as $\eta=\frac{\partial v}{\partial x}-\frac{\partial u}{\partial y}$. Equation (1) can be reduced to yield a second-order equation for the vorticity as follows:

$$
\frac{\partial}{\partial t} \eta-\frac{1}{\operatorname{Re}} \nabla^{2} \eta=\frac{\partial f_{1}}{\partial z}-\frac{\partial f_{3}}{\partial x}
$$

The velocity component equations can be deduced due to $\eta$ and continuity Equation (2):

$$
\nabla^{2} u=-\frac{\partial \eta}{\partial y}, \quad \nabla^{2} v=\frac{\partial \eta}{\partial x}
$$

Fully developed turbulent channel flow is homogeneous in the streamwise direction, and periodic boundary conditions are used in this direction. All unknown quantities are expanded with Fourier series in the streamwise direction and Chebyshev polynomial in the normal direction as follows:

$$
q(x, y, t)=\sum_{m=-N_{1} / 2}^{N_{1} / 2} \sum_{P=0}^{N_{2}} \hat{q}_{m p}(t) \exp \left(i \alpha_{m} x\right) T_{p}(y)
$$

where $\alpha_{m}=m \alpha . \alpha$ is amount of streamwise period, defined as $\alpha=2 \pi / x_{n}, x_{n}$ is the non-dimensional width of computational domain in the streamwise direction; $m$ is streamwise wave number; $T_{p}(y)$ is $p$-order Chebyshev polynomial. 
Substitute the expansions of all unknown quantities into Equations (3) and (4) respectively, and the spectral coefficient equations for each Fourier wave number can be obtained:

$$
\begin{gathered}
\sum_{p=0}^{N_{2}}\left[\frac{\partial}{\partial t}+\frac{1}{\operatorname{Re}}\left(\alpha_{m}{ }^{2}-D^{2}\right)\right] \hat{\eta}_{m p}(t) T_{p}(y) \\
=\sum_{p=0}^{N_{2}}\left[\hat{f}_{1, m p}(t) D-i \alpha_{m} \hat{f}_{2, m p}(t)\right] T_{p}(y) \\
\sum_{p=0}^{N_{2}}\left(-\alpha_{m}{ }^{2}+D^{2}\right) \hat{u}_{m p}(t) T_{p}(y)=-\sum_{p=0}^{N_{2}} \hat{\eta}_{m p}(t) D T_{p}(y) \\
\sum_{p=0}^{N_{2}}\left(-\alpha_{m}{ }^{2}+D^{2}\right) \hat{v}_{m p}(t) T_{p}(y)=\sum_{p=0}^{N_{2}} i \alpha_{m} \hat{\eta}_{m p}(t) T_{p}(y)
\end{gathered}
$$

where differential operator $D$ defines as $D=\frac{d}{d y}$. Equations (5), (6) and (7) are the spectral coefficient formulas for the vorticity, streamwise and normal velocity components.

Boundary conditions of spectral coefficients of vorticity can be derived from the vorticity definition and wall non-slip condition as follows:

$$
\begin{aligned}
& \sum_{p=0}^{N_{2}} \hat{\eta}_{m p}(t)=-\sum_{p=0}^{N_{2}} \hat{u}_{m p}(t) p^{2} \\
& \sum_{p=0}^{N_{2}}(-1)^{p} \hat{\eta}_{m p}(t)=-\sum_{p=0}^{N_{2}} \hat{u}_{m p}(t) p^{2}(-1)^{p-1}
\end{aligned}
$$

Because $\hat{u}_{m p}$ of current time step is unknown, $\hat{\eta}_{m p}$ on the wall can't been solved from (8) directly. In this paper the iteration method is adopted to solve the boundary conditions of $\hat{\eta}_{m p}$ in every time step. The detailed process is as follows:

1) Solve the $\hat{\eta}_{m p}$ on wall from (8) with $\hat{u}_{m p}$ of previous time step.

2) Solve the equation (5) and (6) for $\hat{\eta}_{m p}$ and $\hat{u}_{m p}$.

3) Compare current $\hat{u}_{m p}$ with that of previous time step. If the discrepancy is greater than the given criterion, return to step 1) with current $\hat{u}_{m p}$.

In the total numerical process the most computing time is spent on the FFT and IFFT for spectral method, so this iteration process doesn't remarkably increase the computing time. The computational results indicated that method can provide satisfactory accuracy.

Boundary conditions of streamwise and normal velocitiey-spectral coefficients can be induced similarly:

$$
\sum_{p=0}^{N_{2}} \hat{u}_{m p}(t)=0, \quad \sum_{p=0}^{N_{2}}(-1)^{p} \hat{u}_{m p}(t)=0
$$

$$
\sum_{p=0}^{N_{2}} \hat{v}_{m p}(t)=0, \quad \sum_{p=0}^{N_{2}}(-1)^{p} \hat{v}_{m p}(t)=0
$$

Equations of vorticity-spectral coefficient and velocitiey-spectral coefficients with corresponding boundary conditions compose to respective closed equations sets, which can be solved and the corresponding spectral coefficients can be obtained. The time advancement is carried out by semi-implicit scheme: Crank-Nicolsion for the viscous terms and Adams-Bashforth for the nonlinear terms. The detailed discrete process can be consulted in Reference [14].

\subsection{Computational Model}

Two-dimensional channel flow is chosen as the numerical model. The flow geometry and the coordinate system are shown in Figure 1. The non-dimensional size of computational domain is $[0,2 \pi] \times[-1,1]$. Uniform grids are applied in the streamwise direction and non-uniform grids in the normal direction as follows:

$$
\begin{gathered}
x_{i}=x_{n}(i-1) /\left(N_{1}-1\right) \quad i=1 \ldots N_{1} \\
y_{j}=\cos \left(\theta_{j}\right), \theta_{j}=\pi(j-1) /\left(N_{2}-1\right) \quad j=1 \ldots N_{2}
\end{gathered}
$$

where $x_{n}$ is the non-dimensional width of computational domain in the streamwise direction. $N_{1}$ and $N_{2}$ are the grid numbers in the streamwise direction and normal direction respectively.

\subsection{Small-Perturbation Analysis}

The attenuation of small perturbation in laminar Poiseuille flow and linear growth in the transition process of small perturbation are respectively simulated to prove the accuracy and stability of the proposed algorithm.

The computation is carried out with 4160 grid points $(64 \times 65)$ for a Reynolds number 1500 , which is lower than the transition critical Reynolds number. The time step is 0.001 , which satisfies CFL stability condition. The computation lasts till the solution is steady. The initial flow field is laminar Poiseuille flow with a small

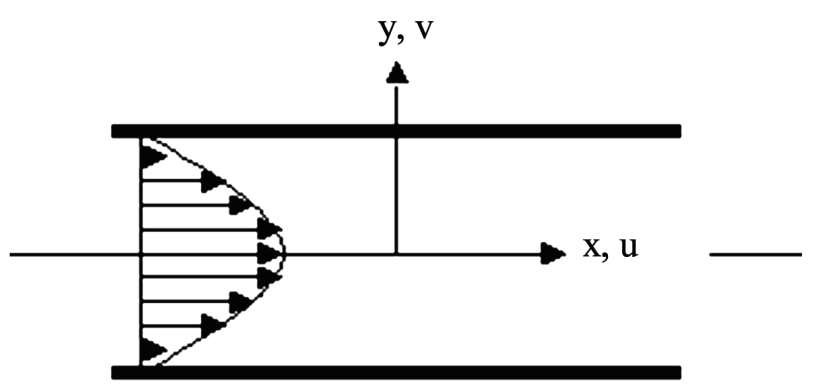

Figure 1. Coordinate system of two-dimensional channel. 
perturbation. Figure 2(a) shows the deviation between initial flow field and Poiseuille flow. Figure 2(b) shows that the profile of steady velocity solution is consistent

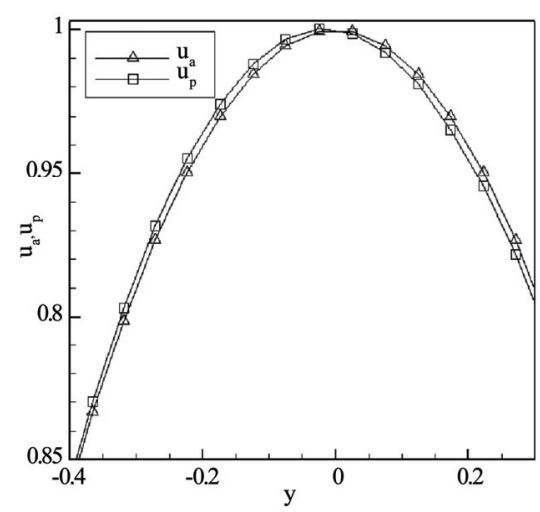

(a)

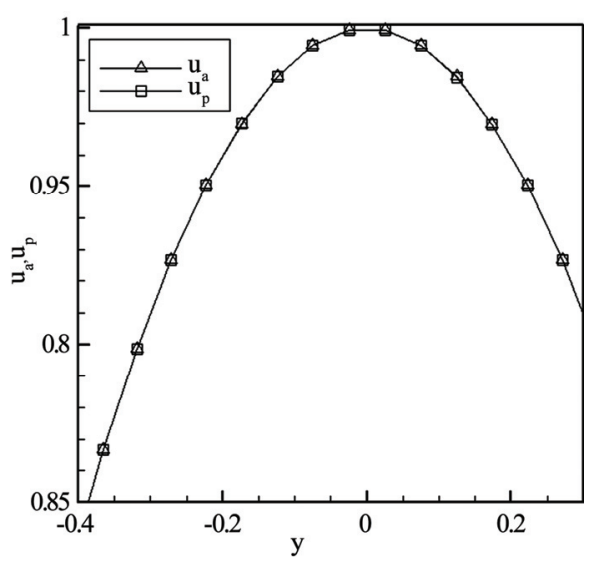

(b)

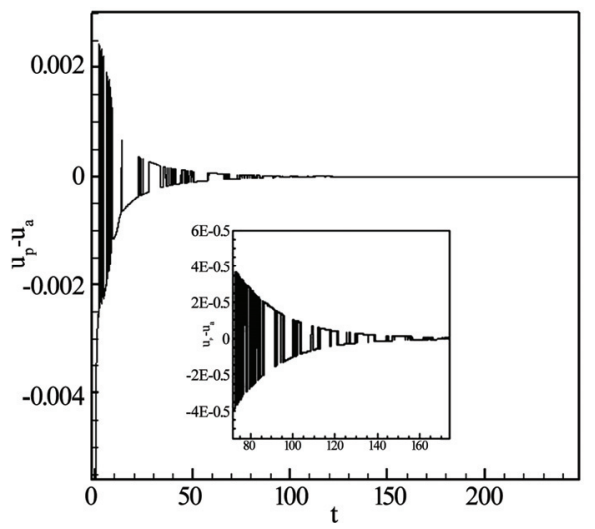

(c)

Figure 2. (a) Contrast between initial velocity profile and Poiseuille profile; (b) contrast between solved steady velocity profile and Poiseuille profile; (c) variation of maximal deviation with time between computational flow field and Poiseuille flow, the figure inside is partial magnification ( $u_{p}$ and $u_{a}$ are the computational streamwise velocity and Poiseuille velocity). with that of Poiseuille flow. Figure 2(c) shows the variation of maximal deviation with time between computational flow field and Poiseuille flow. It can be seen that the maximal deviation gradually reduces and approximately equals zero, even less than $10^{-8}$. Computational results reflect the attenuation of small perturbation in laminar flow and prove the accuracy and stability of the proposed algorithm.

The Reynolds number is increased to 7500 , which is higher than the transition critical Reynolds number. The objective is to calculate the linear growth rate of small perturbation with the proposed algorithm in this paper and compare that with the results by solving the OrrSommerfield equation. The initial flow field with small perturbation is set as follows:

$$
u(x, y)=1-y^{2}+\varepsilon \tilde{u}, \quad v(x, y)=\varepsilon \tilde{v}
$$

where $\tilde{u}$ and $\tilde{v}$ are in accordance with the most instable model of stability theory with the perturbation wave number $\alpha=1.0$ and amplitude $\varepsilon=0.0001$. Kinetic ene- rgy of perturbation is defined as:

$$
E(t)=\int_{-1}^{1} \int_{0}^{2 \pi}\left(\tilde{u}^{2}+\tilde{v}^{2}\right) d x d y
$$

According to the linear theory of small perturbation, the kinetic energy increases exponentially with time, $E(t)=E(0) e^{c t}$. The linear growth rate $c$ is 0.004470 by solving the Orr-Sommerfield equation. Figure 3 shows that the computational results of kinetic energy of perturbation with the proposed algorithm in present paper are in good agreement with the theoretical ones by solving the Orr-Sommerfield equation.

\section{Wall Shear Stress Analysis}

Y. S. Park et al. investigated the effect of periodic blowing and suction on a turbulent boundary layer at three different blowing-suction angles $\left(60^{\circ}, 90^{\circ}\right.$ and $\left.120^{\circ}\right)$

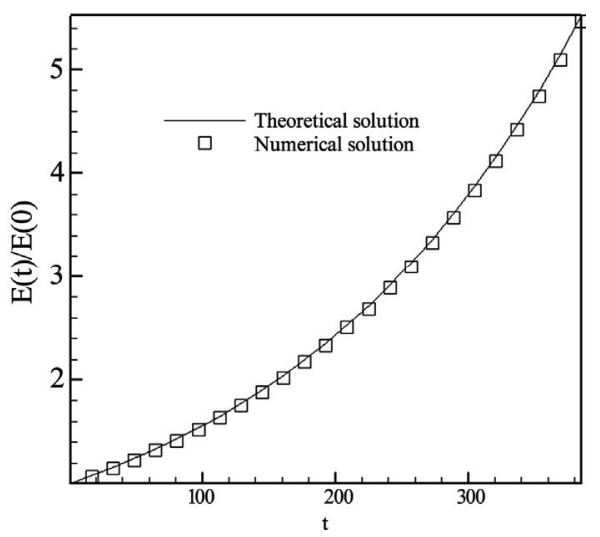

Figure 3. Linear growth of small perturbation. 
with PIV. They found that a better effectiveness of wall shear stress reduction was obtained if the blowing-suction angle was $120^{\circ}$ rather than $90^{\circ}$ [9]. The blowing and suction at this angle changed the streamwise and normal velocities. While the scalar vorticity is defined as $\eta=\frac{\partial v}{\partial x}-\frac{\partial u}{\partial y}$, the vorticity is obviously changed by this control method. So it cam be predicted that the near-wall vortices are also the crucial influence factors to wall shear stress.

A simulation of two-dimensional channel for the Reynolds number 7500 with 8256 grid points $(64 \times 129)$ is carried out to verify this prediction. The initial flow field is Poiseuille flow with the most unstable perturbation which is solved from linear perturbation theory. The turbulence statistics almost no longer change after the non-dimensional time is 600 . The computation is continued for 200 time-steps and the results are as the sample database. The turbulent statistics (such as mean velocity, root-mean-square velocity fluctuations and Reynolds shear stress normalized by friction velocity) are show in Figure 4, which agree well with those in reference [12].

The wall shear stress is defined as $\tau_{w}=\mu \partial u /\left.\partial y\right|_{w}$, where the subscript $w$ denotes the wall. The relation between the vortices above the wall and the wall shear stress can be analyzed with two-point correlation:

$$
R_{1}=\left\langle\frac{\partial u}{\partial y}(x, 0) \eta\left(x, r_{y}\right)\right\rangle
$$

where $r_{y}$ is the spatial distance in $y$ direction and \langle\rangle denotes an average over $y$ and time $t$.

The two-point correlation function is shown in Figure 5. Note that the place $y=1$ is the location of upper wall of the channel. It can be seen that the relation between the near-wall vortices and the wall shear stress really exists. With the increase of spatial distance in $y$ direction, the correlation value rapidly increases to the maximum peak, and then decreases reposefully with the second peak occurring. The spatial distances of the two peaks from the upper channel-wall are 0.03 and 0.2 respectively (i.e. $y=0.97$ and $y=0.8$ ).

A further expression for the relation between near-wall vortices and the wall shear stress can be presented with the instantaneous diagrams of fluctuation velocity vectors. Four successive instantaneous diagrams of fluctuation velocity vectors are shown in Figure 6. It can be clearly seen that a pair of near-wall vortices moves downstream. The $y$-coordinate of the vortices' center A is approximatively 0.8 , which just corresponds to the second peak in Figure 5. This is because the greater vorticity in the center causes the greater two-point correlation value. The streamwise velocity increases from the center to the outside of the near-wall vortices, while it is zero on the wall. So a velocity inflection point occurs in the nearwall region. This can be clearly seen from Figure 7,

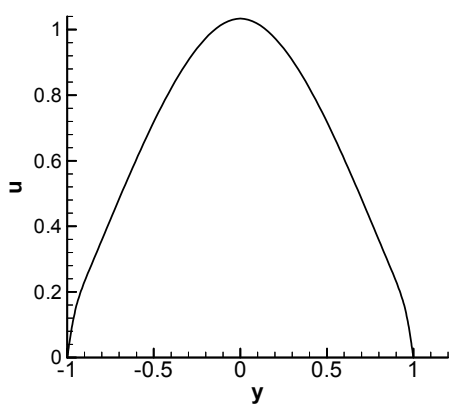

(a)

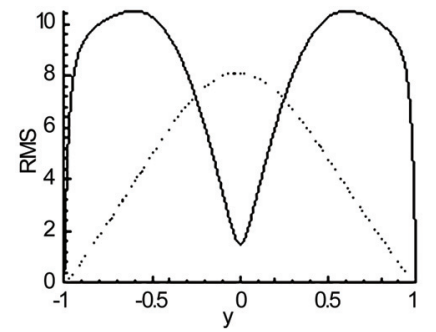

(b)

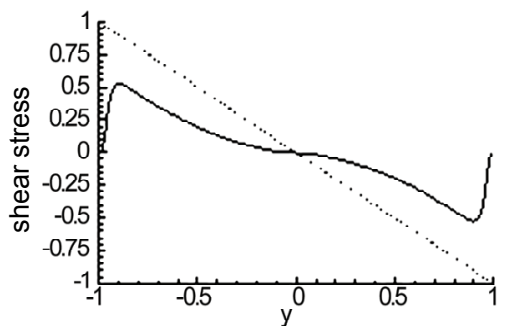

(c)

Figure 4. The turbulent statistics of two-dimensional turbulent channel flow: (a) the mean velocity; (b) the rootmean-square velocity fluctuations (solid line: $\boldsymbol{u}_{r m s}^{\prime+}$; dash line: $v_{r m s}^{\prime+}$ ); (c) Reynolds shear stress and total shear stress (solid line: Reynolds shear stress; dash line: total shear stress).

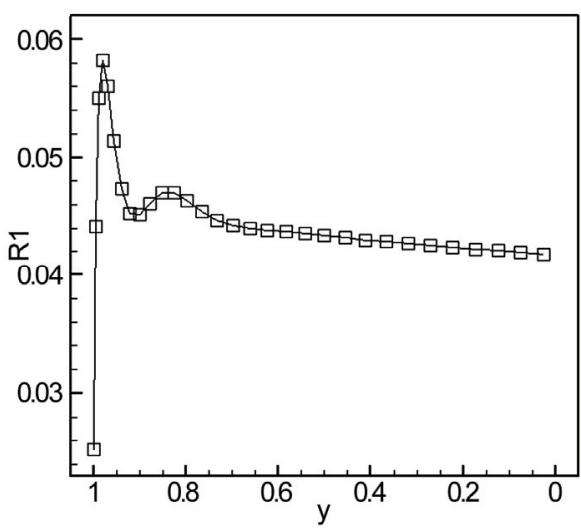

Figure 5. Two-point correlation between the near-wall vorticity and the wall shear rate (where $y=1$ is the location of upper wall of channel). 


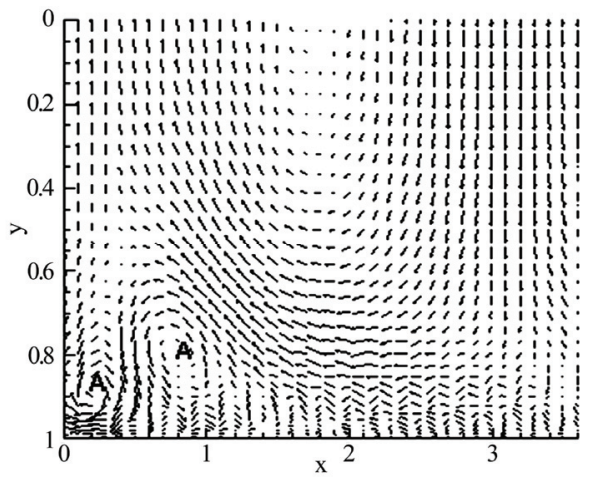

(a)

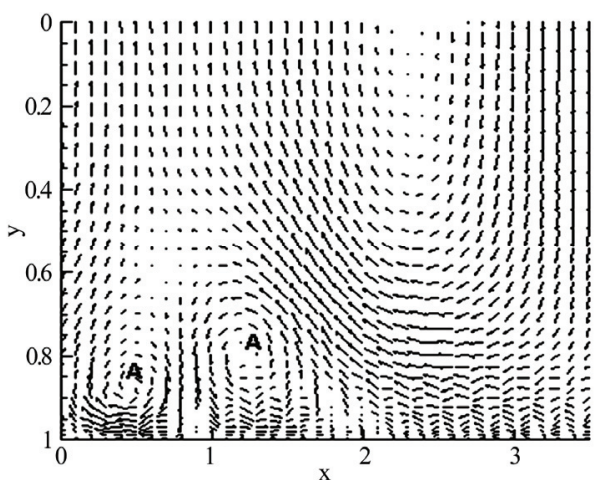

(b)

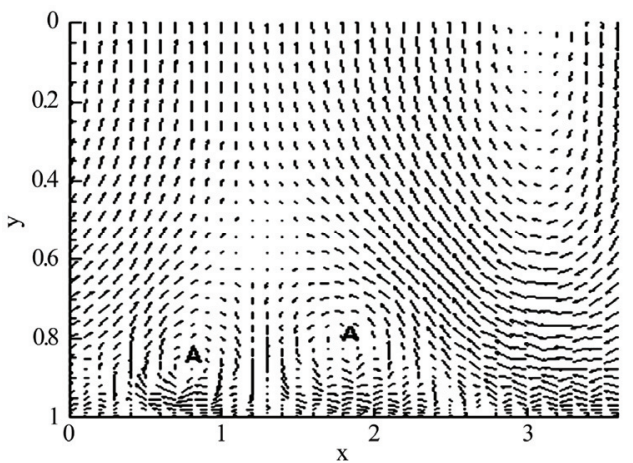

(c)

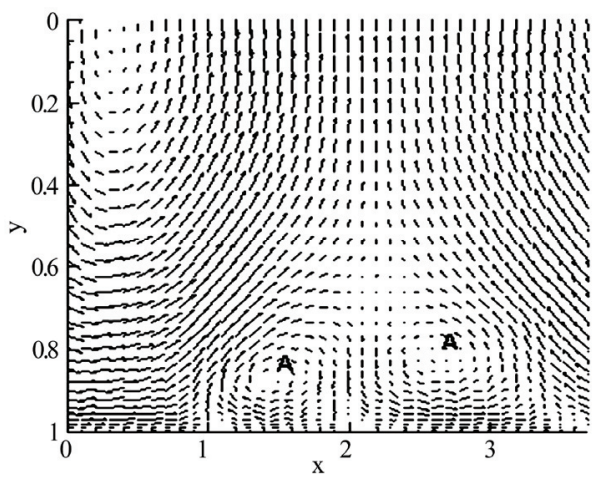

(d)

Figure 6. Instantaneous diagrams of fluctuation velocity vectors. (a) $t=620 ;$ (b) $t=670 ;$ (c) $t=710 ;$ (d) $t=770$.

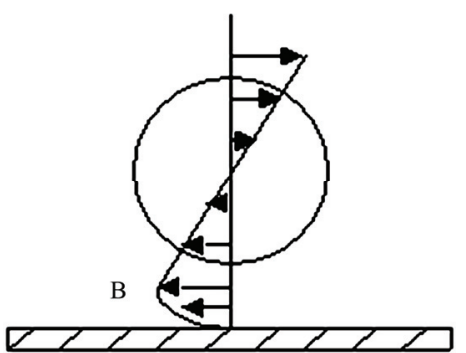

Figure 7. A near-wall vortex model.

where the location B is just the inflection point. It is easy to see that the velocity of the inflection point affects the wall shear rate directly. Therefore the inflection point B just corresponds to the maximum peak in Figure 5.

\section{Conclusions}

A direct numerical simulation of an incompressible twodimensional turbulent channel is performed with spectral method. The numerical results are used to examine the relation between wall shear stress and near-wall vortices. It is found that the wall shear stress is associated with the near-wall vortices and the maximum correlation-value location of near-wall vortices is obtained. It should be pointed out that the three-dimensional simulation has not been carried out due to the shortage of computational resources, but we believe that the qualitative conclusion of near-wall spanwise vortices affecting wall shear stress exists objectively. This achievement is a good supplement to traditional understanding that the wall shear stress is affected by near-wall streamwise vortices. It provides a theoretical guidance for the control of turbulent boundary layers. An optimal control method of turbulent boundary layer may be discovered if the near-wall spanwise vortices are managed to be controlled as well as the streamwise vortices, which is also our research focus for the next step.

\section{Acknowledgments}

We are grateful to Professor J. S. Luo and Dr H. L. Xiao for their help in numerical process. We also thank the financial support provided for this research by National Natural Science Foundation of China (Grant No. 10372033) and Open Foundation of the Key Laboratory of Mechanics on Western Disaster and Environment of the Ministry of Education of China.

\section{References}

[1] J. Shen, E. Malkiel and J. Katz, "Using digital holographic microscopy for simultaneous measurement of 3D 
near wall velocity and wall shear stress in a turbulent boundary layer," Experiment in Fluids, Vol. 45, No. 6, pp. 1023-1035, 2008.

[2] G. Arthur, Kravchenko, H. Choi and P. Moin, "On the relation of near-wall streamwise vortices to wall skin friction in turbulent boundary layers," Physics of Fluid A, Vol. 5, No. 12, pp. 3307-3309, 1993.

[3] E. P. Hammond, T. R. Bewley and P. Moin, "Observed mechanisms for turbulence attenuation and enhancement in opposition-controlled wall-bounded flows," Physics of Fluid, Vol. 10, No. 9, pp. 2421-2423, 1998.

[4] Y. Chang, S. S. Collis and S. Ramakrishnan, "Viscous effects in control of near-wall turbulence," Physics of Fluid, Vol. 4, No. 11, pp. 4069-4080, 2002.

[5] J. Kim, "Control of turbulent boundary layers," Physics of Fluid, Vol. 15, No. 5, pp. 1093-1105, 2003.

[6] J.-I. Choi, C. X. Xu and H. J. Sung, "Drag reduction by spanwise wall oscillation in wall-bounded turbulent flows," AIAA Journal, Vol. 40, No. 5, pp. 842-850, 2002.

[7] K. Kim and H. J. Sung, "DNS of turbulent boundary layer with time-periodic blowing through a spanwise slot," The $5^{\text {th }}$ Aslan computational fluid dynamics conference, Busan, Korea, 30 June-3 July, pp. 1471-1478, 2003.

[8] K.-S. Choi, "Near-wall structure of turbulent boundary layer with spanwise oscillation," Physics of Fluid, Vol. 14, No. 7, pp. 2530-2542, 2002.

[9] Y. S. Park, S. H. Park and H. J. Sung, "Measurement of local forcing on a turbulent boundary layer using PIV,"
Experiments in Fluids, Vol. 34, pp. 697-707, 2004.

[10] S. Chen, G. Eyink and R. E. Ecke, "Physical mechanism of the two-dimensional enstrophy cascade," Physical Review Letters, Vol. 91, pp. 24501, 2003.

[11] W. Kramer, H. J. H. Clercx and G. J. F. van Heijst, "On the large-scale structure and spetral dynamics of twodimensional turbulence in a periodic channel," Physics of Fluids, Vol. 20, pp. 1-15, 2008.

[12] J. Jimenez, "Transition to turbulence in two-dimensional Poiseuille flow," Journal of Fluid Mechanics, Vol. 218, pp. 265-297, 1990.

[13] X. L. Li, Y. W. Ma and D. X. Fu, "High efficient method for incompressiable N-S equations and analysis of twodimensional turbulent channel flow," Acta Mechanica Sinica, Vol. 33, No. 5, pp. 576-586 (in Chinese), 2001.

[14] J. Kim, P. Moin and R. Moser, "Turbulence statistics in fully developed channel flow at low Reynolds number," Journal of Fluid Mechanics, Vol. 177, pp. 133-166, 1987.

[15] L. Kleiser and U. Schumann, "Treatment of incompressibility and boundary layer conditions in 3D numerical spectral simulation of plane channel flows," E. H. Hirschel, Ed., Proceedings of the $3^{\text {rd }}$ GAMM Conference on Numerical Method in Fluid Mechanics, Brunswick, Germany, pp. 165-173, 1980.

[16] Z. W. Hu, C. L. Morfey and N. D. Sandham, "Wall pressure and shear stress spectra from direct simulations of channel flow," AIAA Journal, Vol. 44, No. 7, pp. 15411549,2006 , 